\title{
EchoGéo
}

$35 \mid 2016$

Les découpages territoriaux en Europe

\section{Entre les langues. Textes, voix, circulations. Une sociolinguistique de l'écrit}

Entretien avec Cécile Van den Avenne, réalisé à Paris le 18 décembre 2015

\section{Cécile Van den Avenne et Serge Weber}

\section{(2) OpenEdition}

Journals

Édition électronique

URL : https://journals.openedition.org/echogeo/14478

DOI : $10.4000 /$ echogeo. 14478

ISSN : 1963-1197

Éditeur

Pôle de recherche pour l'organisation et la diffusion de l'information géographique (CNRS UMR 8586)

Référence électronique

Cécile Van den Avenne et Serge Weber, "Entre les langues. Textes, voix, circulations.

Une sociolinguistique de l'écrit », EchoGéo [En ligne], 35 | 2016, mis en ligne le 19 avril 2016, consulté le

11 août 2021. URL : http://journals.openedition.org/echogeo/14478 ; DOI : https://doi.org/10.4000/ echogeo.14478

Ce document a été généré automatiquement le 11 août 2021.

EchoGéo est mis à disposition selon les termes de la licence Creative Commons Attribution - Pas d'Utilisation Commerciale - Pas de Modification 4.0 International (CC BY-NC-ND) 


\title{
Entre les langues. Textes, voix, circulations. Une sociolinguistique de l'écrit
}

\author{
Entretien avec Cécile Van den Avenne, réalisé à Paris le 18 décembre
} 2015

\author{
Cécile Van den Avenne et Serge Weber
}

1 - Serge Weber (SW). Pour prendre au sérieux l'intitulé même de cette rubrique, «Sur l'Écrit ", vous avez répondu à mon invitation pour ce numéro - et je vous en remercie vivement - car en tant que linguiste, ou plutôt sociolinguiste, vous travaillez précisément sur le statut de l'écrit. À chaque fois que nous avons eu l'occasion d'évoquer vos travaux, que ce soit sur Marseille ou au Mali, vos deux terrains principaux, j'ai remarqué une évidente convergence avec des pratiques de recherche en géographie. En effet, vous faites un véritable travail de terrain, proche de l'ethnographie, comme de nombreux géographes, et vous avez justement collaboré avec des géographes dans vos missions, ce qui vous a rendu leurs méthodes familières. De plus, vous interrogez un certain nombre de thématiques centrales pour les géographes: la migration, la toponymie vernaculaire, le repérage, les approches postcoloniales, les formes locales des rapports sociaux... Il est certain que vos terrains sur l'opération d'adressage de Mopti, sur les lettres d'auditeurs d'une radio locale ou sur le plurilinguisme éclaireront les géographes qui s'interrogent sur les rapports entre la langue et la géographie. Je vous propose donc de nous en dire davantage sur l'importance de la langue et en particulier de la langue écrite dans la réflexion sur les représentations de l'espace et les pratiques spatiales.

2 - Cécile Van den Avenne (CVA). Je suis sociolinguiste. «Sociolinguistique » aujourd'hui est une étiquette qui regroupe des chercheurs que n'unifient ni leurs méthodologies, ni leur objet, ni leur cadre théorique, mais qui ont pour point commun de s'intéresser aux dimensions sociales des pratiques linguistiques, et surtout qui considèrent tous qu'à travers les pratiques linguistiques se construisent rapports de force et inégalités sociales. Lorsque je dis "sociolinguistique », c'est pour préciser le regard je porte sur 
des phénomènes linguistiques, l'intérêt qui me guide, la démarche que je cherche à suivre : une approche intégrée des faits linguistiques et sociaux. Et en ce sens je me sens proche du courant de la sociolinguistique critique initié par Monica Heller, et qui a des affinités avec les travaux de Josiane Boutet, c'est-à-dire une sociolinguistique qui "met au centre de ses préoccupations non pas la langue comme système, ni même la société comme système ou comme structure, mais la langue comme partie inhérente des pratiques sociales " (Boutet, Heller, 2007, p. 312). La cohérence première de mes travaux, qui rend compte aussi de mon inscription disciplinaire, est fournie par les objets que j'étudie et la méthodologie que je mets en œuvre. Les objets sur lesquels je travaille depuis plus de dix ans sont des objets textuels écrits: de la plaque de rue au roman, de la lettre manuscrite envoyée à une radio au manuel de langue, ce sont des objets issus d'un processus d'entextualisation, au sens de Silverstein et Urban (1996), de mise en texte qui, pour certains, les met en circulation, les décontextualisant de leurs conditions de production.

3 Et mon travail consiste à analyser ces textes pour en donner des clés de compréhension, en tâchant de les recontextualiser, de reconstruire leurs conditions de production, de réception, de circulation. Je considère ensemble tout genre de textes, du plus modeste, pris dans des usages quotidiens, du plus populaire, circulant dans une sphère de consommation plus ou moins large, aux plus reconnus et légitimes. Je propose ainsi une "sociolinguistique des textes", dont le cadrage est fourni notamment par les travaux de l'anthropologue Karin Barber (2007). La particularité des textes est leur double existence, à la fois comme dépendant contextuellement d'un acte de langage et comme entité autonome dans l'espace social. Et si cette double existence des textes intéresse l'anthropologue, pour ce qu'elle lui permet de comprendre des relations sociales, des idées et valeurs d'une culture qui a produit ces textes, elle est aussi au cœur de ce que l'on peut appeler une approche sociolinguistique des textes, qui rend compte de leur matérialité linguistique et textuelle, et des sens contextuels qu'ils vont prendre selon les sphères dans lesquels ils circulent et sont consommés.

4 - SW. La première fois que je vous ai rencontrée, en 2004, votre expérience de collecte de choses dites et de choses écrites par des anonymes à Marseille m'avait interpelé. Café Verre, la revue à la fois poétique, artistique et ethnographique à laquelle vous collaboriez, me semblait déjà une expérimentation qui ne pouvait que passionner les géographes. Dans votre travail de linguiste, vous avez une préoccupation qui me semble constante pour l'espace, en particulier les espaces urbains, et un regard qui rappelle fortement le regard géographique.

5 - CVA. Café Verre a été un projet collectif auquel j'ai participé, et qui a été pour moi une façon d'entrer dans la ville de Marseille, à un moment où je m'y installais. Il s'agissait essentiellement d'un travail d'édition reposant sur une collecte préalable, de « choses entendues", "papiers trouvés", mots écrits sur les murs, un travail de recueil photographique et d'entretiens. Tout a été déposé à la bibliothèque de Marseille ${ }^{1}$. C'est à Marseille que j'ai rencontré des géographes et tout d'abord Elisabeth Dorier. Nous avons fait du terrain ensemble, à Mopti, au Mali, je vais en reparler, et elle m'a introduite dans un groupe qu'elle a constitué avec Philippe Gervais-Lambony. Nous avons fait plusieurs workshops, travaillant sur la notion de citadinité, à Marseille, mais aussi Johannesburg et Istanbul. De cette collaboration est né un livre collectif, Vies citadines, dans lequel j'ai rédigé le chapitre intitulé «Parler » (Dorier-Aprill, GervaisLambony, 2007). Il s'agissait de proposer un cadre où envisager la ville à travers les 
pratiques langagières qui la tissent, comme une des façons d'appréhender les vies citadines. Cela permettait, me semble-t-il, de s'éloigner de, ou du moins relativiser, les analyses de la ville mettant l'accent sur l'affaiblissement actuel des liens citadins et la «fragmentation». Je pointais différents phénomènes comme les fabrications de langues que génère la vie urbaine, la tension entre la pratique identitaire de langues manière de consolidation de l'entre-soi en ville - et les pratiques véhiculaires de langues - fonctionnant comme ciment de l'appartenance citadine et comme compétence acquise en ville de passer d'un code linguistique à l'autre en changeant d'espace ou de groupe. Ceci permet de rendre compte de la ville en train de se faire. Le chapitre était enrichi d'encadrés écrits par plusieurs membres du collectif, à partir de leurs propres terrains.

6 - SW. Votre thèse portait sur Marseille, mais plutôt sur des questions de migrations et de plurilinguisme...

7 - CVA. Quand j'ai fait ma thèse, que j'ai soutenue en $1999^{2}$, j'ai recueilli et analysé des biographies migratoires et langagières de locuteurs d'origine malienne vivant tous, au moment de l'enquête, depuis vingt ou trente ans à Marseille. Tous pratiquaient en France deux langues, le français et le bambara. Pour certains de ces locuteurs, le bambara (langue véhiculaire au Mali) n'était pas langue première, c'est la langue cependant qu'ils pratiquent en France avec leurs compatriotes, et qu'ils ont transmis à leurs enfants. Ces Maliens sont des gens très plurilingues. Or ce plurilinguisme s'est réduit au moment de l'arrivée en France. Je citerai l'exemple de l'un d'entre eux, un Dogon qui faisait en sorte, avec un bon bagage socioculturel puisqu'il était médecin, de ne jamais parler bambara au Mali et qui, arrivant à Marseille dans la toute petite communauté des Maliens de Marseille, s'est remis au bambara car il y était bien obligé. De même, il y avait une famille minyanka qui avait totalement abandonné l'usage du minyanka car ils étaient les seuls à parler cette langue à Marseille. On peut donc dire qu'il y a une sur-utilisation véhiculaire du bambara en migration, pour ceux dont ce n'était pas la première langue. La migration a changé diachroniquement leur pratique du plurilinguisme.

8 J'ai exploré la façon dont on met en récit sa propre migration, dont on met en mots ses appartenances linguistiques et identitaires, des positions difficiles à exprimer. Les analyses laissent apparaître notamment la complexité des positions entre ici et là-bas, et la complexité de la position du chercheur, pris dans cette relation post-coloniale vécue par des personnes migrantes issues de l'ex-métropole française, devenue société d'accueil.

9 - SW. Vous souhaitiez pour cet entretien nous relater votre expérience d'arpenteuse d'une ville malienne, pour évoquer la question du langage dans une opération d'adressage...

10 - CVA. Le travail que je vais évoquer, je l'ai conduit pour l'essentiel avec Aïssatou Mbodj-Pouye ${ }^{3}$, anthropologue chargée de recherches au CNRS à l'IMAF ${ }^{4}$ - qui est aussi mon laboratoire de rattachement secondaire. Aïssatou Mbodj-Pouye a travaillé pour sa thèse sur les pratiques de littératie (literacy) ${ }^{5}$ des agriculteurs d'une zone de la région cotonnière de Fana (Mali), qui présente la particularité suivante. Cette région a connu une alphabétisation des adultes en bambara dans les années 1960 et des projets pilotes de scolarisation en français et en bambara, dont le but était d'être diffusé dans l'ensemble du pays. Elle travaillait sur ce terrain et était tombée sur des cahiers personnels que tenaient certains agriculteurs où ils notaient tout un tas de choses. Ces 
cahiers sont intéressants parce qu'ils avaient la particularité d'être plurilingues. Elle ne disposait pas d'outils balisés pour décrire cela. Elle a donc débarqué dans mon bureau avec des photos de ces cahiers (illustration 1). Rien qu'à les regarder, je les trouvai beaux: des listes de courses dans les deux langues, des listes de semailles... Cela confortait mon goût des papiers trouvés. On a donc commencé à travailler ensemble, moi en tant que sociolinguiste et elle comme socio-anthropologue.

Illustration 1 - Extrait du corpus d'Aïssatou Mbodj-Pouye

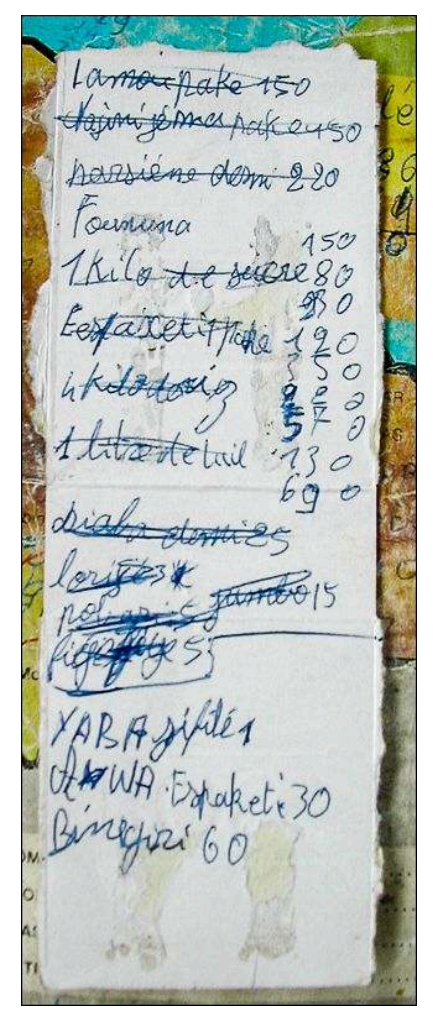

Auteur : Aïssatou Mbodj-Pouye.

11 Ce que révélaient ces cahiers renvoie à tout un courant de la sociolinguistique, autour de ce qu'on appelle le " code switching», ou " alternance codique ", sur des productions orales, émanant de personnes plurilingues qui passent d'une langue à l'autre à l'oral. C'est plus récemment qu'on s'y intéresse dans des productions écrites, alors que cela existe dès l'Antiquité. Nous avons publié un premier article dans Langage et société, en proposant un outil d'analyse de ce type de production (Mbdoj-Pouye, Van den Avenne, 2007). Il nous fallait reprendre un certain nombre de schémas descriptifs pour l'oral et voir ce qui fonctionnait pour l'écrit en tenant compte de ses spécificités, en particulier la mise en page (selon les langues). Par exemple, ces cahiers révélaient des procédés très graphiques: les locuteurs de bambara écrivent cette langue avec une graphie en «script» (minuscules d'imprimerie), car c'est comme cela qu'ils sont alphabétisés alors que l'écriture du français est apprise en cursives. Certains utilisent la couleur verte pour écrire en arabe - car il y a de l'arabe aussi, notamment pour les textes religieux. Dans l'espace du cahier, nous observions une mise en colonne des deux langues principales, des petits lexiques, des données d'état civil. Notamment les dates de naissance des différents enfants: date officielle en français, en bambara pour le calendrier lunaire traditionnel, par exemple, en particulier pour des pratiques d'ordre 
magico-religieux. Travailler ensemble était vraiment passionnant. Or, j'avais déjà fait un premier terrain à Mopti en 1999 avec la géographe Elisabeth Dorier, qui y démarrait un nouveau terrain après avoir longtemps travaillé au Congo-Brazzaville, suivi d'un second en 2000-2001.

Ce premier terrain mené avec Elisabeth Dorier relevait d'une commande ponctuelle: elle travaillait sur des problèmes d'assainissement, de gestion des eaux et des déchets, dans une ville, Mopti, qui s'est construite sur des remblayages d'ordures. Elle avait besoin de cartographier la ville car il n'existait pas de carte à jour depuis la période coloniale. Plus encore, il n'y avait aucun adressage des rues: les gens utilisaient des toponymes vernaculaires. C'est ce dont j'ai été chargée: effectuer le repérage toponymique. J'ai donc arpenté la ville avec elle, je l'ai regardée faire, avec une carte ancienne, notant de multiples détails, j'interrogeais les gens pour noter les toponymes vernaculaires. Les noms de rue à Mopti s'appellent Karé, «lieu». Par exemple «Fali Karé », « l'endroit des ânes», ou des noms de rue avec la famille la plus importante comme « Tapo Karé » par exemple.

13 Après, nous avons travaillé sur d'autres choses à Mopti, notamment sur des histoires de "connivences citadines", c'est le titre de l'article que nous avons écrit ensemble (Dorier-Aprill, Van den Avenne, 2001). Pour expliciter ce terme, je dirais que c'est une sorte de "vivre ensemble ", pour reprendre une expression à la mode, propre à cette ville très plurilingue et multiethnique marquée par de multiples couches historiques. Je me suis fondée sur des récits urbains, les légendes de fondation urbaine, qui partent toutes d'une justification d'alliances entre grandes familles: la famille qui tient la mosquée, celle qui tient la pêche et la gestion de l'eau, les familles de forgerons... Ces légendes rendent compte d'une spécialisation spatiale de quartiers qui s'organisent autour de ces familles-là. Cet ancrage a l'air très ancien, alors que la ville de Mopti à proprement parler, telle qu'elle existe aujourd'hui avec ses quartiers sur remblais, est une création coloniale !

14 Cela m'a donné une connaissance de Mopti particulièrement précise, je connaissais toutes les rues, les placettes, l'arpentage, les grandes familles, les enjeux fonciers. C'est une ville qui est très restreinte dans son accroissement spatial du fait des zones inondables, l'extension est donc progressive et se fait par l'accumulation d'ordures. Et ceci suppose toute une organisation pour établir qui va remblayer et où.

15 - SW. Avez-vous travaillé sur d'autres corpus écrits lors de ces terrains? Vous aviez évoqué, avant l'entretien, un animateur radio et des lettres fascinantes qu'il recevait, narrant des rêves à interpréter. Ces pistes m'ont frappé par l'originalité de leur apport ethnographique.

16 - CVA. Cette expérience m'a donné envie de retourner à Mopti, j'ai donc proposé à Aïssatou d'y faire un terrain avec elle. La dernière fois, en 2001, je m'étais retrouvée à la radio locale Radio Jamana, qui avait plusieurs antennes dans le pays. On m'avait montré des lettres en bambara, envoyées par un auditeur assidu qui écrivait très fréquemment des lettres à cette radio. J'ai voulu le rencontrer, on m'a donc dit de communiquer à la radio pour lancer un appel. C'était un éleveur agriculteur peul d'un petit village du cercle de Mopti, à une trentaine de kilomètres, qui écoutait la radio comme on fait beaucoup au Mali (la radio est un lien important avec le monde urbain et l'extérieur). Je l'ai donc rencontré, nous avons fait un bref entretien. Il ne parlait pas français, sa première langue était le peul. Adolescent, il avait suivi une campagne d'alphabétisation en bambara, qui était donc la seule langue qu'il savait écrire, et il s'en servait pour 
écrire. Sur les usages de l'écrit, sur ce que font les gens de l'écriture, quand ils savent écrire, c'était particulièrement intéressant. À quoi cela leur sert-il, au-delà des campagnes d'alphabétisation? En effet, ces campagnes, dites "fonctionnelles» ont pour but de permettre aux agriculteurs de lire des notices, faire leurs comptes, rembourser les emprunts etc., mais il y a un relatif silence sur ce que les gens peuvent en faire d'autre, par exemple écrire des lettres, des pensées personnelles etc.

Nous avons donc fait plusieurs terrains centrés sur Radio Jamana, autour des écrits des auditeurs. Notamment nous nous sommes liées avec un animateur qui avait une émission d'interprétation des rêves. Il est très polyglotte : il parle le songhay ${ }^{7}$, le peul, le bambara, le français, l'arabe. Il est aussi enseignant d'arabe au lycée - au Mali il y a un deuxième cursus en arabe dans le secondaire.

Illustration 2 - Radio Jamana, les archives

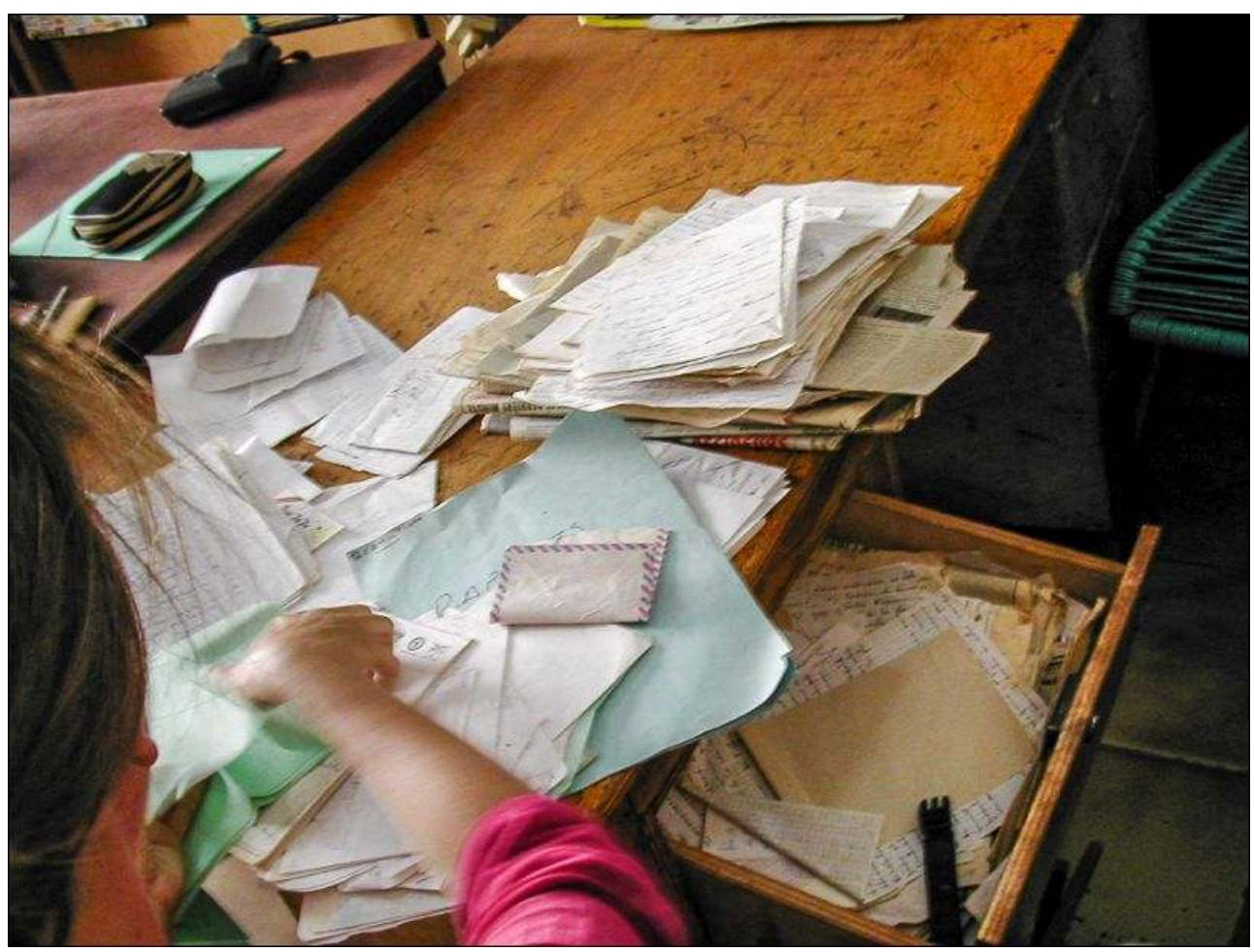




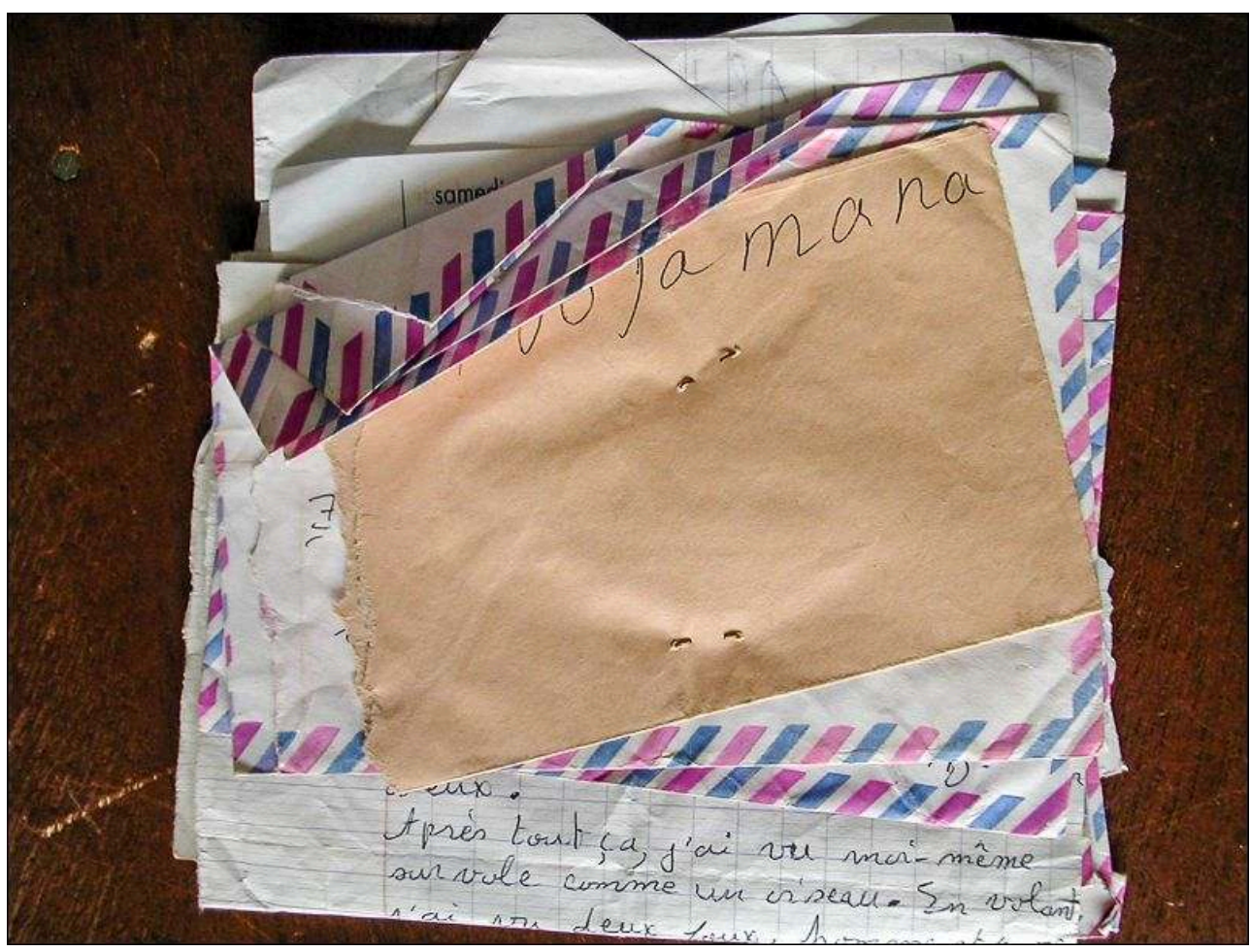

Auteur : Cécile Van den Avenne.

Quand nous lui avons parlé de notre projet, il nous a ouvert les tiroirs de ses archives, qui contenaient des liasses de lettres (illustration 2). Il avait une organisation à la fois très précise et désordonnée. Il faisait l'interprétation des rêves à l'antenne. Pour ce faire, il recevait les lettres, travaillait dessus en amont, avec une clef des songes musulmane. L'interprétation n'a rien à voir avec nos idées occidentales: l'interprétation des rêves se fait terme à terme, mot à mot: un animal vu en songe correspond par exemple à une personne ou à une notion. On peut dire que ce sont des mots-images. Et lui, il décodait. Ce qui veut dire que la question de la traduction est très importante. La clef est en arabe, c'est un texte imprimé qui se vend couramment. Les lettres sont dans les autres langues. Il pointe les mots clefs et va les traduire en arabe : c'est d'une grande complexité, ce qu'il fait, du point de vue linguistique. Nous avons commencé à explorer ce qu'il nous a donné : ce corpus de trois cents lettres archivées (elles étaient agrafées après chaque émission et stockées dans un grand tiroir qu'il a vidé devant nous dans un grand sac plastique), dans plusieurs langues. Nous en avons tiré un premier article publié récemment dans le Journal des Africanistes (Mbodj-Pouye, Van den Avenne, 2013). Cela prouvait que des personnes, dotées de compétences linguistiques qu'on pourrait dire à première vue réduites, parviennent à écrire des choses assez efficaces pour permettre de raconter un rêve qui soit interprétable. Sonia Branca $(1989,1994)$ a écrit sur les écrits des gens peu lettrés sous la Révolution française, qui donnent lieu à des formes d'écriture qui sont du point de vue de la norme très malhabiles, peu standardisées, mais qui ont une efficacité indéniable. C'est ce que nous avons voulu montrer, à travers la manière d'utiliser les deux langues (le français ou le bambara, ou les deux langues), ainsi que l'arabe et le peul - dont il est utile de rappeler qu'il s'écrit depuis très longtemps notamment, en ajami, la graphie arabe qui a été utilisée depuis l'islamisation, soit les X-XII siècles. 
19 Nous avons publié des choses ponctuelles, mais nous n'avons pas pu y retourner depuis 2008, à cause de la guerre, malgré notre envie de travailler avec notre animateur Nous avons eu quelques contacts téléphoniques depuis le début du conflit, et nous avons appris qu'il a servi de traducteur pour les forces militaires de la coalition internationale. Ce qui nous fait nous demander par quel biais il a été sollicité : comment quelqu'un avec des compétences comme les siennes s'est-il retrouvé à faire ce travail ? - il y aurait une monographie à faire sur lui.

20 - SW. Ce que vous évoquez me fait penser à la fois aux usages de la littératie qu'avait explorés Richard Hoggart et la question de la raison graphique développée par Jack Goody. Ces auteurs vous-ont-ils inspirée?

21 - CVA. J'aime beaucoup le travail de Hoggart. C'est lui qui a introduit le terme de « littératie » dans les sciences humaines et sociales. Les travaux de Jack Goody ensuite ont été très importants dans ce champ, depuis la parution de La raison graphique en 1977 (Goody, 1979 [1977]). C'est le premier à rendre compte du fait que l'introduction de l'écriture dans une société change un certain nombre de procédures, même cognitives : quand on met en liste, qu'on utilise des supports mémoriels etc. Il s'est ainsi attaché à dépasser la question du "grand partage " entre les sociétés avec et sans écriture, à casser cette frontière figée par l'anthropologie, même celle de Claude Lévi-Strauss, en travaillant justement sur des terrains africains, notamment au Ghana. Les conceptions de Goody ont depuis été largement discutées, notamment l'idée que la littératie puisse être abordée comme un phénomène autonome, que globalement l'introduction de pratiques de l'écrit aurait partout les mêmes effets cognitifs et sociaux. Après Goody un certain nombre de chercheurs ont montré au contraire qu'il fallait étudier ces pratiques de l'écrit de façon contextualisée, prises dans des rapports de pouvoir particuliers par exemple.

22 - SW. Pourriez-vous nous en dire plus sur votre travail en cours, Administrer par l'écrit?

23 - CVA. À l'époque où nous avons commencé à travailler avec Aïssatou, elle participait à un groupe de recherche sur l'anthropologie de l'écriture, dirigé par Béatrice Fraenkel, anthropologue de l'écrit, et directrice de recherches à l'EHESS, au sein d'un programme ANR intitulé "Écologies et politiques de l'écrit " , avec des terrains très différents, dans les périodes historiques et dans l'espace. Elle nous avait proposé d'y participer, comme nous avions commencé le terrain de Mopti et qu'il y avait un projet sur la toponymie vernaculaire. Entretemps, l'adressage de la ville de Mopti avait été fait car il y avait eu une injonction de la Banque mondiale pour que les villes africaines prennent en charge l'adressage de la ville, dans une optique de «bonne gouvernance » selon ses termes, pour repérer les habitants, les recenser, prélever l'impôt de manière plus systématique. Cette injonction a été assortie de financements et de l'installation d'une agence à Bamako. L'adressage a donc été fait pour Bamako et certaines villes secondaires sur un modèle d'adressage fourni par l'agence, un modèle américain. Cela impliquait d'établir un quadrillage numéroté d'est en ouest, qui correspondait assez bien au plan des villes coloniales. Mais il fonctionnait moins bien dans les quartiers plus auto-construits, où l'adressage a été fait comme s'il y avait une ligne droite, sans entrer dans le détail.

24 Nous avons retracé la manière dont les autorités ont procédé à l'adressage (qui venait justement d'être fait) et la manière dont les habitants se le sont appropriés. Le terrain a consisté à rencontrer différents acteurs qui ont participé à l'opération d'adressage puis d'autres qui sont directement concernés, dans leur pratique professionnelle notamment, par cette mise en place de l'adressage. Nous avons donc travaillé avec la 
direction de la voirie, qui nous a recommandé à un agent voyer, lequel nous a emmenées dans la ville avec un odomètre, un instrument sur roue qu'on fait rouler sur le sol et qui sert à calculer une distance. Il l'utilisait pour attribuer une numérotation métrique des portes des habitations. Nous avons essayé de comprendre comment on avait procédé pour les cas problématiques comme une porte murée, une autre ouverte sur un autre mur. Cela révélait des choses intéressantes sur la gestion de l'espace privé par rapport à l'espace public: la frontière n'est pas si nette. Et nous avons suivi un certain nombre d'acteurs qui avaient utilisé ce nouvel adressage. J'ai notamment fait une tournée avec un releveur de compteurs de l'électricité du Mali - ce qui a épargné des coupures d'électricité à certains ménages !

il connaissait les ruelles par cœur, mais dans les nouveaux quartiers, même avec l'adressage, il se perdait et moi aussi je n'y comprenais rien. Je me souviens qu'on a beaucoup tourné dans un quartier neuf pour trouver une rue dont on avait le numéro, on trouvait la rue qui précédait ou suivait numériquement, mais pas celle-là. La municipalité avait tenu aussi à ce que certaines rues aient un nom : a priori l'objectif était simplement administratif, ce nom n'était même pas censé être utilisé par les habitants, qui utilisaient encore l'ancien système vernaculaire. Quand la municipalité a nommé des rues, dans certains cas elle a réutilisé des noms vernaculaires qui ne posaient pas de problème. Mais quand elle a décidé de prendre des noms de notables, les habitants n'étaient pas toujours d'accord. Certains noms pouvaient leur poser des problèmes, pour des questions de querelles entre familles. Cela révélait donc un double adressage: d'un côté un système qui contrôlait, et de l'autre un système qui fonctionnait dans l'appropriation par les habitants.

Suite à ce projet, nous avons d'abord rédigé un rapport' ${ }^{9}$, que nous avons notamment remis à la direction de la voirie de Mopti qui l'a archivé, puis un chapitre d'un ouvrage collectif dirigé par Béatrice Fraenkel qui est toujours à paraitre ${ }^{10}$.

Nous sommes allées voir également des archives municipales. C'est un voyage en soi, un lieu assez poétique mais un cauchemar pour le chercheur (illustration 3). Nous avons trouvé des comptes rendus de conseils municipaux qui dataient de la décolonisation, mais déjà les habitants avaient exprimé leur désaccord avec les propositions de noms de rues. Par exemple, "avenue de l'Indépendance » était relativement consensuel, de même pour des grands noms de notables anciens. Mais lorsque les familles étaient repérables, cela ne leur semblait pas légitime.

EchoGéo, 35 | 2016 
Illustration 3 - Archives municipales de Mopti

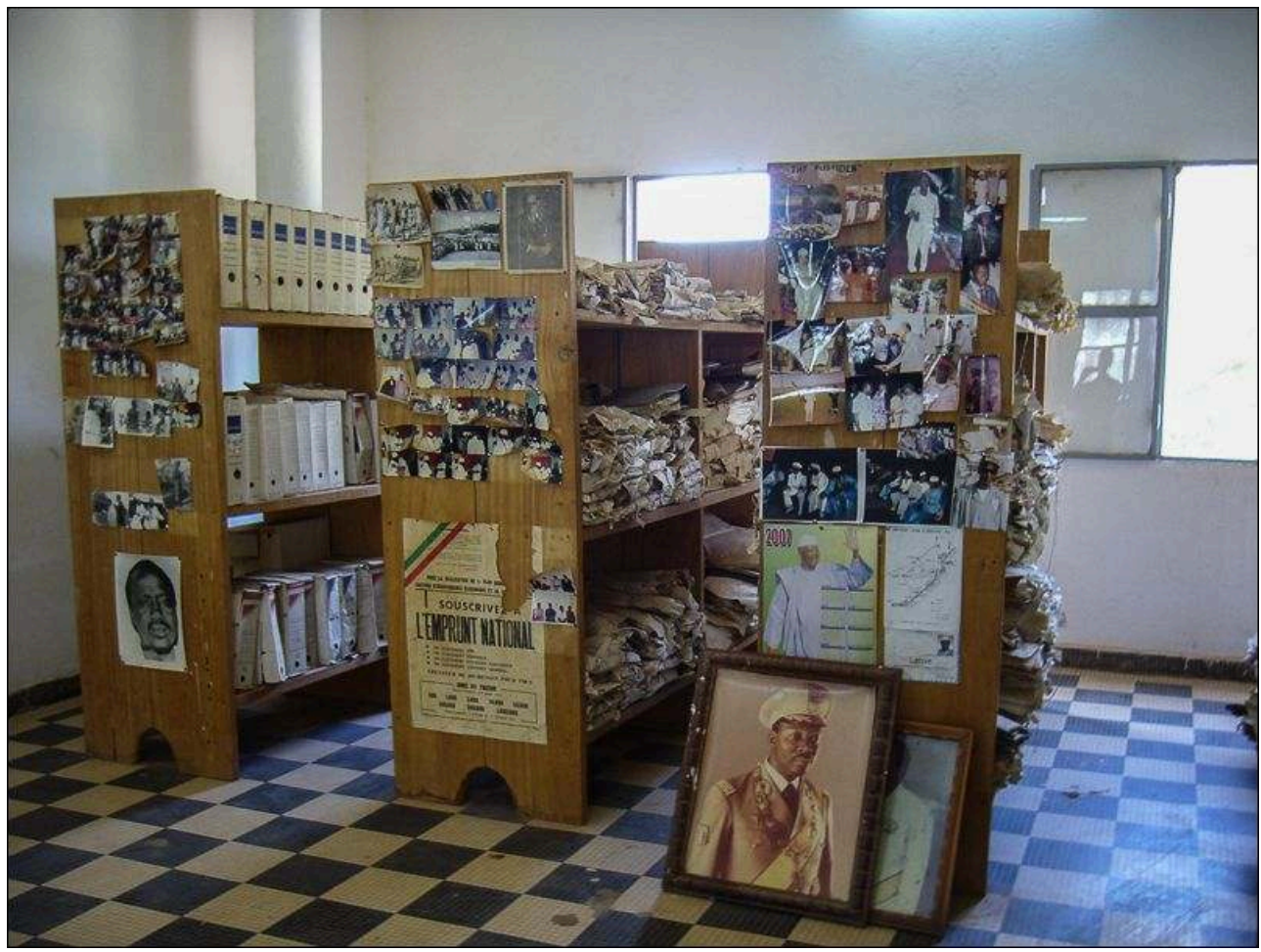

Auteur : Cécile Van den Avenne. plaque dans les rues, cela consacre certaines familles, ce qui pose un problème. Ce n'est pas pour rien que sur certaines plaques de noms de rues, les noms des notables ont été grattés (illustration 4) 
Illustration 4 - Rue de Mopti

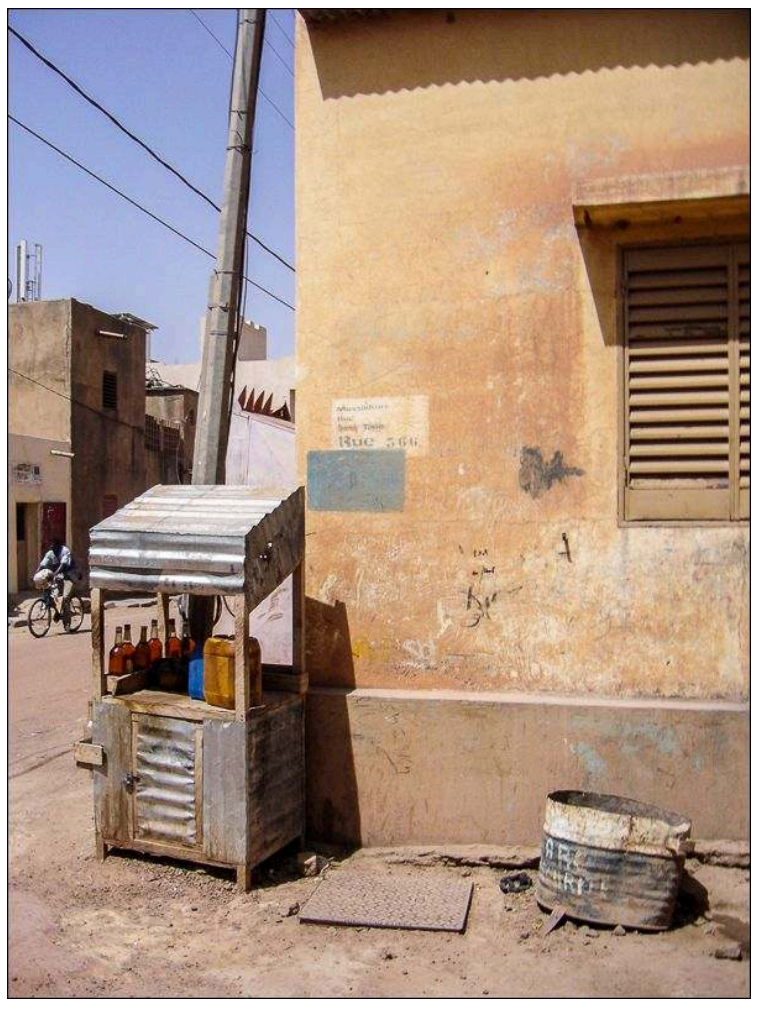

Auteur : Cécile Van den Avenne.

31 - SW. On peut donc dire que vous avez un goût certain pour ce type d'écrits, un peu fragiles?

32 - CVA. C'est vrai. Par exemple, la première fois qu'Aïssatou Mbodj m'a montré ses cahiers, j'ai trouvé que c'étaient des objets vraiment beaux, pas seulement passionnants scientifiquement, ils me touchaient aussi. La ville de Mopti est construite majoritairement en banco, en terre crue, et elle est pleine d'écrits, de graffiti, qui s'effacent avec les saisons. J'ai beaucoup photographié et, notamment de façon exhaustive, les écrits d'un «fou » (on me l'a décrit comme tel) qui parcourait la ville et écrivait des textes entiers sur les murs (illustration 5). Je ne sais pas forcément encore quoi en faire, cela fait écho en tout cas à d'autres pratiques non scientifiques que j'ai depuis longtemps, et notamment depuis Café Verre... 
Illustration 5 - Sur les murs de Mopti

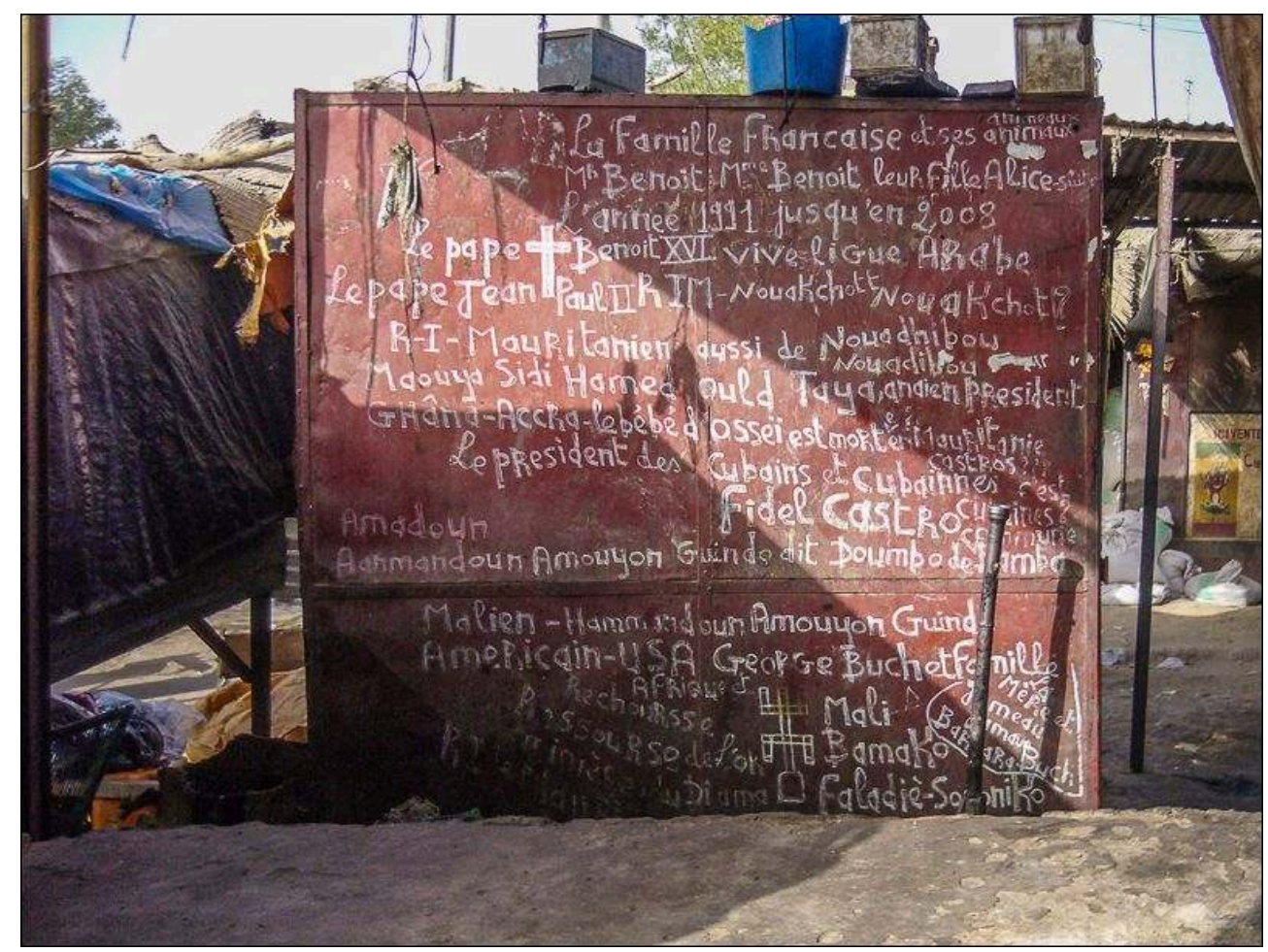

Auteur : Cécile Van Den Avenne.

\section{BIBLIOGRAPHIE}

Barber K., 2007. The Anthropology of Texts, Persons and Publics. Oral and written culture in Africa and beyond. Cambridge, Cambridge University Press.

Boutet J., Heller M., 2007. Enjeux sociaux de la sociolinguistique : pour une sociolinguistique critique. Langage et société, 3/2007, nº 121-122, p. 305-318.

Branca-Rosoff S., 1989. Vue d'en bas : des écrits "malhabiles" pendant la période révolutionnaire. Langage et société, vol. 47, $\mathrm{n}^{\circ} 1$, p. 9-27.

Branca-Rosoff S., Schneider N., 1994. L'écriture des citoyens. Une analyse linguistique de l'écriture des peu-lettrés pendant la période révolutionnaire. Paris, Klincksieck.

Dorier-Apprill E., Gervais-Lambony P. (dir.), 2007. Vies citadines. Paris, Belin.

Dorier-Apprill E., Van Den Avenne C., 2001. La connivence citadine et ses exclus. Le cas de Mopti, ville moyenne du Mali. Annales de la recherche urbaine, $\mathrm{n}^{\circ} 90$ “ Les seuils du proche ", p. 117-125.

Goody J., 1979 [1977]. La raison graphique. La domestication de la pensée sauvage. Paris, Minuit.

Mbodj-Pouye A., 2013. Le fil de l'écrit. Une anthropologie de l'alphabétisation au Mali. Lyon, ENS éditions. 
Mbodj-Pouye A., Van den Avenne C., 2007. « C'est bambara et français mélangé ». Analyser des écrits plurilingues à partir du cas de cahiers villageois recueillis au Mali. Langage et société, $\mathrm{n}^{\circ} 120$, p. 99-127.

Mbodj-Pouye, A., Van den Avenne, C., 2013. Faire entendre sa voix : deux corpus de lettres envoyées à une radio locale à Mopti. Journal des Africanistes, vol. 83, nº 1, p. 38-69.

Silverstein M., Urban G. (dir.), 1996. Natural histories of discourse. Chicago, University of Chicago Press.

Van Den Avenne C., Dorier-Apprill E., 2002. Usages toponymiques et pratiques de l'espace urbain à Mopti (Mali). Marges Linguistiques, $n^{\circ}$ 3, mai 2002. http://marges.linguistiques.free.fr/

Van den Avenne C., 2004. Changer de vie, changer de langues. Paroles de migrants entre le Mali et Marseille. Paris, L'Harmattan.

\section{NOTES}

1. http://documentsdartistes.org/artistes/bonnet/repro1.html.

2. Une version retravaillée de cette thèse a abouti à la publication d'un ouvrage (voir Van den Avenne C, 2004).

3. Nous nous sommes rencontrées quand elle finissait sa thèse à Lyon avec Bernard Lahire. Elle a publié un ouvrage issu de sa thèse (Mbodj-Pouye, 2013).

4. Institut des Mondes Africains. Il s'agit d'une Unité mixte de recherche interdisciplinaire (histoire ancienne et contemporaine, anthropologie, science politique, archéologie) dont les travaux portent sur l'ensemble du continent africain. Il a été créé au 1er janvier 2014, par la fusion de trois laboratoires : le Centre d'études des mondes africains (CEMAf), le Centre d'études africaines (CEAf) et le Centre d'histoire sociale de l'islam méditerranéen (CHSIM).

5. On entend par littératie l'ensemble des savoirs et des pratiques individuelles et collectives liées à l'écrit (écriture et lecture). Voir notamment La littératie. Autour de Jack Goody, Pratiques $\mathrm{n}^{\circ}$ 131-132, dossier coordonné par J.-M. Privat \& M. Kara, décembre 2006, Metz.

6. Nous avons rendu compte de ce travail de recueil toponymique dans un article, voir Van Den Avenne C., Dorier-Apprill E., 2002.

7. Le songhay (sous différentes formes dialectales) est une langue importante (le nombre de locuteurs est estimé à 3 millions), parlée au Nord du Mali et au Niger principalement. Les locuteurs de songhay représenteraient environ $8 \%$ de la population malienne (plus d'un million de locuteurs). Le peul (sous différentes formes dialectales également) est une langue de très grande diffusion (les estimations vont au-delà de 60 millions de locuteurs) parlée dans plusieurs pays d'Afrique de l'Ouest et d'Afrique centrale. Le peul du Mali, ou fulfuldé, est parlé principalement dans le Macina, par plus d'un million de locuteurs. Le bambara au Mali est à la fois la langue première de la majorité de la population et une langue véhiculaire à l'échelle du pays.

8. Programme développé par le laboratoire Traitement et Communication de l'Information (CNRS-ENST) et l'équipe Anthropologie de l'écriture du IIAC (CNRS-EHESS).

9. On peut le trouver en ligne sur ma page personnelle: http://www.van-den-avenne.net/ recherche.

10. Mbodj-Pouye, A., Van den Avenne, C. (à paraître), L'opération d'adressage à Mopti. Administrer par l'écrit, administrer l'écrit. In Jean-Charles Depaule, Béatrice Fraenkel (dir.), Ecritures urbaines : les lois de la ville, Editions MSH-Unesco, collection "Les mots de la ville". 


\section{AUTEURS}

\section{CÉCILE VAN DEN AVENNE}

Cécile Van den Avenne, cecile.vandenavenne@ens-lyon.fr, est maître de conférences HDR en sociolinguistique à l'ENS-Lyon.

\section{SERGE WEBER}

Serge Weber, serge.weber@gmail.com, est maître de conférences HDR en géographie, urbanisme et aménagement à l'Université Paris-Est Marne-la-Vallée. 